This is an electronic reprint of the original article. This reprint may differ from the original in pagination and typographic detail.

Author(s): Isoniemi, Tommi; Tuukkanen, Sampo; Cameron, David C.; Simonen, Janne; Toppari, Jussi

Title: $\quad$ Measuring optical anisotropy in poly(3,4-ethylene dioxythiophene):poly(styrene sulfonate) films with added graphene

Year: $\quad 2015$

Version:

Please cite the original version:

Isoniemi, T., Tuukkanen, S., Cameron, D. C., Simonen, J., \& Toppari, J. (2015).

Measuring optical anisotropy in poly(3,4-ethylene dioxythiophene):poly(styrene sulfonate) films with added graphene. Organic electronics, 25(October), 317-323. https://doi.org/10.1016/j.orgel.2015.06.037

All material supplied via JYX is protected by copyright and other intellectual property rights, and duplication or sale of all or part of any of the repository collections is not permitted, except that material may be duplicated by you for your research use or educational purposes in electronic or print form. You must obtain permission for any other use. Electronic or print copies may not be offered, whether for sale or otherwise to anyone who is not an authorised user. 


\title{
Measuring optical anisotropy in poly(3,4-ethylene dioxythiophene):poly(styrene sulfonate) films with added graphene
}

\author{
Tommi Isoniemi ${ }^{1}$, Sampo Tuukkanen*2, David C. Cameron ${ }^{\dagger 3}$, Janne Simonen ${ }^{1}$, and J. Jussi Toppari ${ }^{\ddagger 1}$ \\ ${ }^{1}$ Nanoscience Center, Department of Physics, P.O.Box 35 (YN), FI-40014 University of Jyväskylä, Finland \\ ${ }^{2}$ Department of Electronics and Communications Engineering, Tampere University of Technology (TUT), \\ P.O.Box 692, FI-33101 Tampere, Finland \\ ${ }^{3}$ Miktech Oy, Sammonkatu 12, FI-50130 Mikkeli, Finland
}

September 9, 2015

\begin{abstract}
Graphene is a 2D nanomaterial having a great potential for applications in electronics and optoelectronics. Composites of graphene with conducting polymers have shown high performance in practical devices and their solution-processability enables low-cost and high-throughput mass manufacturing using printing techniques. Here we measure the effect of incorporation of graphene into poly(3,4-ethylene dioxythiophene):poly(styrene sulfonate) (PEDOT:PSS) to the optical anisotropy, absorbance and conductivity of the film. Uniaxial anisotropy in PEDOT:PSS films has been thought to be caused by the spin-coating process used in fabrication. We have characterized spray- and spincoated films using ellipsometry and total internal reflection spectroscopy, the latter especially for films too thick and uneven for ellipsometry, and show that spray-coating, similar to inkjet printing, also produces consistently anisotropic properties even in very thick and uneven films. Possible plasmonic excitations related to graphene are not seen in the films. The optical and electrical anisotropy of graphene/PEDOT:PSS enables routes to high performance devices for electronics, photonics and optoelectronics.
\end{abstract}

\section{Introduction}

Nanostructured carbon materials, such as graphene $[1,2]$ and carbon nanotubes (CNT) [3], have gathered vast interest due to numerous desirable properties such as high and especially tunable electrical and thermal conductivity, high tensile strength, high surface area, chemical sensitivity, flexibility, transparency and light weight. Their potential has been already proven in the fields of electronics

\footnotetext{
* Present address: Department of Automation Science and Engineering, Tampere University of Technology (TUT), P.O.Box 692, FI-33101 Tampere, Finland.

$\dagger$ Present address: R\&D Center for Low-Cost Plasma and Nanotechnology Surface Modification, Masaryk University, Kotlářská 2, CZ-61137 Brno, Czech Republic.

$\ddagger$ Corresponding author (J.J. Toppari). E-mail address: j.jussi.toppari@jyu.fi
}

$[1,4,5]$, optoelectronics and optics $[5,6,7]$, materials technology $[8,9]$ as well as in energy technology and biotechnology [10]. More recently CNTs and graphene in particular have also been of interest due to their plasmonic properties $[11,12]$.

Printing technologies offer a promising route for low-cost and high-throughput manufacturing of flexible, lightweight and even transparent electronic devices such as flexible displays, radio frequency identification (RFID) antennas, batteries, supercapacitors and solar cells $[13,14]$. In addition, the combination of printing technologies with disposable, non-toxic organic molecular materials offers a route towards green electronics [15]. The nanostructural carbon materials and their composites with polymers are promising solution-processable materials, e.g., for supercapacitor electrodes [16, 17].

Poly(3,4-ethylene dioxythiophene) (PEDOT) is a low band-gap conjugated conducting polymer with a high charge mobility and good stability. It has a poor solubility, but when poly(styrene sulfonate) (PSS) is used as a counterion, it forms a stable dispersion that can be used to produce films with high conductivity and good transparency in the visible region. ([18], p. 113) Due to these properties it has found widespread usage, particularly as a transparent electrode material and as an antistatic agent [18].

It has been shown that spin-coated PEDOT:PSS films display anisotropic properties, both optically as a uniaxial refractive index as well as in their electrical conductivity. The conductivity in the direction parallel to the film can be 500 times higher than perpendicular to the film ([18], p. 133). These anisotropic features have been explained as being caused by PSS-rich regions lying parallel to the film [19]. Electrostatic mist deposition also produces anisotropic PEDOT:PSS thin films, even before baking [20]. The choice of substrate, especially the surface roughness, has a significant effect on the structure of PEDOT:PSS thin films. Uniaxial anisotropy is stronger when the deposition has been done on a very smooth surface such as crystalline silicon [21]. 
The optical anisotropy is a highly desired feature in PEDOT:PSS based optoelectronic devices such as polymer based light-emitting diodes and photovoltaic devices [22]. For instance, the photovoltaic performance of crystalline silicon/organic heterojunction solar cells was enhanced by increased anisotropy of PEDOT:PSS film [23].

Due to their enhanced electronic and optical properties, graphene and graphene/polymer composite films are promising materials for photonic and optoelectronic devices [6], for example ultra-fast lasers [24]. Graphene/PEDOT:PSS has been used as low-cost counter electrode material for high performance dye-sensitized solar cells (DSSC) [25]. Very recently, large scale synthesis of graphene/PEDOT:PSS was demonstrated using in situ polymerization and the composite was used for the fabrication of thermoelectric and DSSC devices for energy harvesting applications [26].

Graphene/PEDOT:PSS is a promising alternative also for conventionally used transparent and conductive coatings such as commercial indiumtin-oxide (ITO) films. Further, the solution processability of graphene/PEDOT:PSS allows for low-cost and high throughput fabrication making it interesting material for flexible and transparent optoelectronic devices such as a flexible display, solar cell and sensors. For example, printed graphene/PEDOT:PSS films have been used as stretchable [27] and transparent [28, 29] electrodes.

Despite all of the above, optical properties of conductive polymers incorporating graphene have not been widely studied, even though optical characterization and analysis of these composites is a prerequisite to be able to design high performance optoelectronic devices. Here, we present analysis of optical properties of a graphene/PEDOT:PSS composite using ellipsometry and reflection measurements in total internal reflection conditions. The obtained results are compared with results from bare PEDOT:PSS films. In addition, the results of reflectance measurements are compared with the results from simulations based on experimental data from the literature.

Films that are thick, highly absorbing or uneven are not favorable for ellipsometry. However, angledependent spectroscopy in total internal reflection (TIR) conditions can provide a stronger and more reliable signal in these cases. TIR absorption spectroscopy is usually used to measure absorption for a very small amount of material, because the evanescent wave at the TIR interface only reaches about $100 \mathrm{~nm}$ beyond the interface, and the spectroscopic signal comes from this region. When used on a thin metal film in plasmonics, this setup is also referred to as the Kretschmann configuration. It thus reveals simultaneously the interference patterns as well as

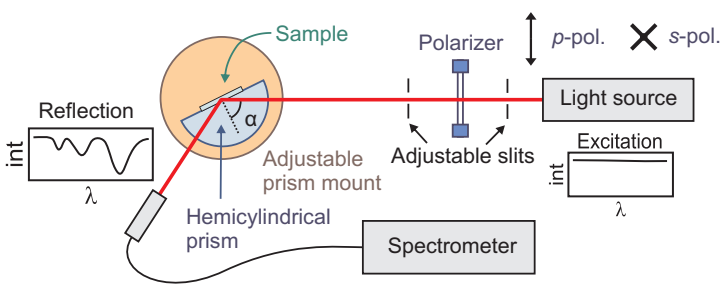

Figure 1: Schematics of the total internal reflection setup and geometry used in the experiment.

possible plasmonic excitations.

The setup can also be used when the TIR conditions are not completely met, and in our case we determine anisotropy from the difference of $s$ and $p$-polarization in reflectance. Measuring the reflectance with different polarizations of incoming light yields information about the anisotropy of the film due to its influence on thin-film interference [30]. This has usually been used in the case of an anisotropic film with perpendicular beam incidence, when it is known as reflection anisotropy spectroscopy [31].

\section{$2 \quad$ Experimental}

\subsection{Solution-processable materials}

Two different solution processable materials were used for the fabrication of thin films using spin- and spray-coating methods. PEDOT:PSS ink (Clevios PH500 solution purchased from H.C. Starck GmbH) is an aqueous dispersion with the PEDOT:PSS ratio of 1:2.5 by weight. The product is a blue dispersion of PEDOT:PSS containing organic solvents and polymeric binders with a solid content of 1.0-1.3 wt\%. The PEDOT:PSS ink viscosity is $8-25 \mathrm{mPas}$.

PEDOT:PSS/graphene ink (Phene+ I3015 transparent inkjet conductive ink purchased from Innophene Co., Ltd.) is an aqueous dispersion containing 1-5 wt-\% PEDOT:PSS polymer, 1-5 wt-\% graphene, 5-10 wt- $\%$ diethylene glycol and 1-5 wt- $\%$ ethanol. The PEDOT:PSS/graphene ink has a solid content of $0.6 \mathrm{wt}-\%$, its viscosity is $7.2 \mathrm{mPas}$ and the conductivity is given as $300 \mathrm{~S} / \mathrm{cm}$. The graphene flakes are homogeneously dispersed in the polymer matrix [27]. For some PEDOT:PSS/graphene samples ultrasonication (Elma S120H Elmasonic 1000 W, 90 minutes) was used on the ink before applying it on the substrate. This was done to check for any possible effects resulting from breaking down the graphene flakes within the ink.

\subsection{Preparation of films}

The PEDOT:PSS and PEDOT:PSS/graphene films were produced with a spray-coating process. Glass 
substrates (Präzisions Glas \& Optik Selected White Float glass, $20 \times 20 \times 1.25 \mathrm{~mm}, n=1.52$ ) were cleaned with acetone and isopropanol. . The spraycoating was done using a Silverline airbrush perpendicularly on the substrate at a distance of $10 \mathrm{~cm}$ operated with standard compressed (1 bar) air. The substrate was on a hot-plate at $115{ }^{\circ} \mathrm{C}$, and the spray-coating was done slowly to avoid the formation of any visible droplets. For comparison, some samples were instead spin-coated at 2000 or $6000 \mathrm{rpm}$. Deposited PEDOT:PSS films were baked in an oven at $100{ }^{\circ} \mathrm{C}$ for $6 \mathrm{~min}$ and PEDOT:PSS/graphene films at $120^{\circ} \mathrm{C}$ for $10 \mathrm{~min}$. The samples were stored in ambient conditions.

\subsection{Characterization of the films}

The sheet resistances of the thin films were obtained with a four-point probe measurement setup. The probe included four electrodes with mercury contacts in a line with probe separation of $2.5 \mathrm{~mm}$. A Keithley 2456 Sourcemeter was used to drive a constant current of $0.1 \mathrm{~mA}$ (except $1 \mu \mathrm{A}$ for samples with square resistances over $100 \mathrm{k} \Omega$ ) to the outer electrodes and to measure the voltage at the inner electrodes. Five measurements were averaged for each sample, using the standard error of the mean, and the sheet resistances were calculated depending on the dimensions of the samples [28].

Thickness and topography of the films were characterized using a Veeco Dimension 3100 atomic force microscope (AFM). Scanning electron microscopy (SEM) was done on cut surfaces of the polymer films on a silicon substrate with a Raith e-Line 50 system.

The optical reflection measurements were performed in a TIR configuration depicted in Fig. 1. Measured incidence angles ranged from $13^{\circ}$ to $74^{\circ}$, extending across the TIR angle of $41^{\circ}$ for a simple glass-air interface. The glass slides with polymer layers on top were mounted on a flat face of a hemicylindrical prism with index matching oil (Zeiss Immersol 518F, $n=1.518$ ). The angle of the collimated excitation light (220 W Oriel tungstenhalogen lamp) was tuned with a goniometric prism mount, and the reflected light was collected to an optical fiber and fed to a spectrometer (Jobin Yvon iHR320) equipped with a CCD camera (Jobin Yvon Symphony CCD-1024X256-OPEN-STE).

Absorbance measurements were done with a PerkinElmer Lambda 850 UV/VIS Spectrometer. The transmission data were normalized in respect to the empty glass substrate, and the absorbance is presented as the average absorption at wavelength range 400-700 $\mathrm{nm}$.

Variable-angle spectroscopic ellipsometry (VASE) measurements were done with a J.A. Woollam M- (a)

(c)

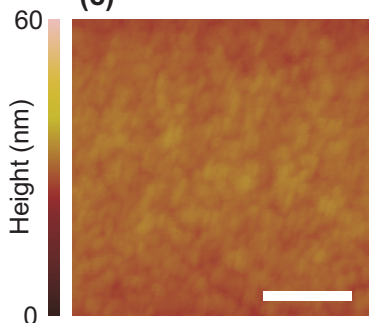

(e)

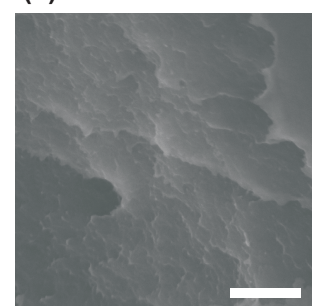

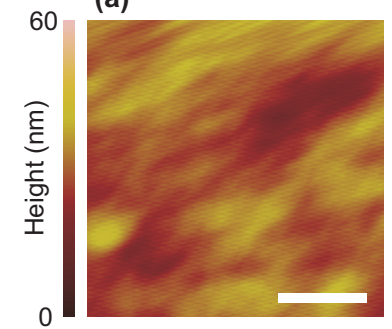

(b)

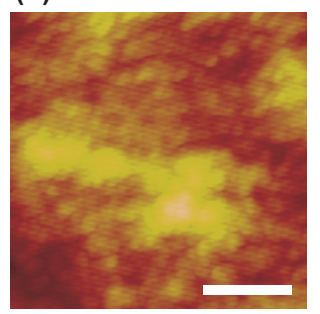

(d)

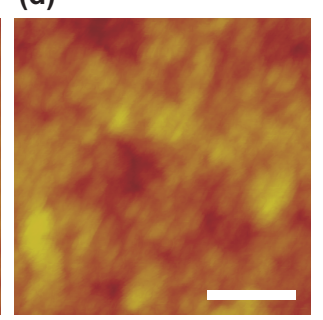

(f)

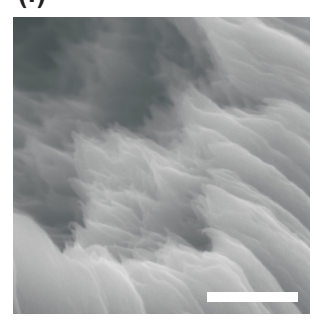

Figure 2: AFM micrographs of flat surfaces of spray-coated films of (a) $2100 \pm 100 \mathrm{~nm}$ thick PEDOT:PSS, root mean square roughness for the image area $r_{q}=4.5 \mathrm{~nm}(\mathrm{~A}-9)$ and (b) $2150 \pm 150 \mathrm{~nm}$ thick PEDOT:PSS/graphene, $r_{q}=5.7 \mathrm{~nm}$ (B-9), and corresponding spin-coated films of (c) $29 \pm 1 \mathrm{~nm}$ thick PEDOT:PSS, $r_{q}=0.96 \mathrm{~nm}(\mathrm{~A}-\mathrm{S} 1),(\mathrm{d})$ $34 \pm 5 \mathrm{~nm}$ thick PEDOT:PSS/graphene, $r_{q}=3.1 \mathrm{~nm}$ (B-S1). SEM micrographs at $5 \mathrm{kV}$ acceleration voltage of cut surfaces of (e) PEDOT:PSS and (f) PEDOT:PSS/graphene. All scale bars are $300 \mathrm{~nm}$.

2000UI ellipsometer. As the measured films were on glass substrates, the second glass interface was covered with black paint to eliminate reflections from it.

\section{Results and discussion}

\subsection{Film morphology and conductivity}

Details of the fabricated samples, including sample names/codes (starting with 'A' for PEDOT:PSS films, 'B' for PEDOT:PSS/graphene films and 'C' for PEDOT:PSS/graphene films with presonication), film thickness $d$, root mean square roughness $r_{q}$ in a $1 \mu \mathrm{m} \times 1 \mu \mathrm{m}$ area and sheet resistance $R_{S}$, are presented in Table S1 in Supplementary material. The spray-coated films are typically about ten times thicker than spin-coated films $(340-2100 \mathrm{~nm}$ versus $29-77 \mathrm{~nm}$ ). 
AFM images of typical spray- and spin-coated films are presented in Figs. 2a,b and 2c,d, respectively. It can be noticed that the PEDOT:PSS/graphene films have typically higher roughness than bare PEDOT:PSS films, which is particularly noticeable in spin-coated films. This can result from the presence of graphene flakes which could prevent relaxation of the ink surface during the deposition process.

Spray-coated samples expectedly have a much higher roughness than the spin-coated ones, especially when considering large areas. Because film thickness measurements were done with AFM on one $10 \mu \mathrm{m}$ long cut interface for each film, and the uncertainty is estimated from this measurement, the thickness values for a whole spray-coated sample may have even higher variations than those presented.

SEM images of cut edges of droplet-deposited polymers are presented in Figs. 2e and 2f. The presence of flakes can be observed in the PEDOT:PSS/graphene film. However, some layered edge formation also appears in the bare PEDOT:PSS film.

The addition of graphene into the PEDOT:PSS film had a clear effect on the properties of the film. The most remarkable effect was observed in the conductivity. For instance, a $550 \mathrm{~nm}$ thick PEDOT:PSS/graphene film has a sheet resistance of $61 \pm 12 \Omega / \mathrm{sq}$, which is less than $1 \%$ of the sheet resistance of a similar bare PEDOT:PSS film, i.e., $10.8 \pm 1.2 \mathrm{k} \Omega / \mathrm{sq}$ (see Table $\mathrm{S} 1$ and Fig. $\mathrm{S} 1$ in Supplementary material). Spray-coated bare PEDOT:PSS had a conductivity at $0.8 \pm 0.3 \mathrm{~S} / \mathrm{cm}$, while PEDOT:PSS/graphene had a conductivity of $310 \pm 40$ S/cm. PEDOT:PSS/graphene with 90 minutes of sonication had an unchanged conductivity of $320 \pm 60 \mathrm{~S} / \mathrm{cm}$. The conductivities were determined by linear fits for films of different thicknesses (Fig. S3 in Supplementary material). As expected, there is a linear dependence between increasing film thickness and increasing conductivity in the deposited films. However, in spray-coated PEDOT:PSS, this linear dependence is not very clear. Thin spin-coated samples have lower conductivities, 0.104 $\pm 0.013 \mathrm{~S} / \mathrm{cm}$ for PEDOT:PSS (29 and $77 \mathrm{~nm}$ thick films) and $130 \pm 30 \mathrm{~S} / \mathrm{cm}$ for unsonicated PEDOT:PSS/graphene (34 nm thick film).

\subsection{Absorption measurements}

It can be noticed that the optical absorption increases linearly with the thickness of the film and decreases as a function of sheet resistance (see Fig. S2 and S4 in Supplementary material). The absorbance used here is defined in section 2.3. (roughly comparable to absorbance at $550 \mathrm{~nm}$ ) and representative transmittance spectra are shown in Fig. S8 of the Supplementary material. Fitting a linear dependency on absorption as a function of thickness one obtains an absorption per film thickness of $0.46 \pm 0.051 / \mu \mathrm{m}$ for spray-coated PEDOT:PSS, while PEDOT:PSS/graphene had a clearly lower value at $0.27 \pm 0.031 / \mu \mathrm{m}$. Sonicating PEDOT:PSS/graphene for 90 minutes had no clear effect on this, producing films with $0.28 \pm 0.051 / \mu \mathrm{m}$ absorption. For spin-coated samples the absorption values were somewhat lower, $0.38 \pm 0.031 / \mu \mathrm{m}$ for two PEDOT:PSS samples and $0.14 \pm 0.031 / \mu \mathrm{m}$ for one (not sonicated) PEDOT:PSS/graphene sample.

The optical absorbance in a similar PEDOT:PSS/graphene ink has been shown to have a linear relationship with graphene content [32]. In light of this, the measured higher absorbance of plain PEDOT:PSS is surprising, but the compositions of our measured inks are not directly comparable.

\subsection{Reflection measurements}

Results from the reflection measurements are shown in Fig. 3 for some of the samples (more data in Supplementary material in Figs. S5-S7). Clear interference patterns are visible in all of the samples, and with both polarizations. The anisotropic optical properties of the films are clearly visible as different positions and depths of the destructive interference resonances at $s$ - and $p$-polarizations. In addition, inclusion of graphene enhances the resonances and anisotropy between the polarizations, and sonication does so even further, as more clearly visible in Figs. S5, S6 and S7. However, in case of the sonication, stronger interference resonances are most likely due to smoother film surfaces.

Collective optical excitations with angle-dependent resonances that cannot be explained by interference were found on thin films of single-walled metallic carbon nanotubes [12], but not seen in PEDOT:PSS or PEDOT:PSS/graphene films. The lack of this kind of plasmonic behaviour, which might suppress optical signals, increases their viability for optoelectronics applications. The optical excitation in TIR conditions can easily reach through a $\mu \mathrm{m}$-scale PEDOT:PSS/graphene film, as can be seen in Fig. S7f of the Supplementary material. In this case, surface plasmon polaritons (SPP) were excited on a $50 \mathrm{~nm}$ gold film through a $1280 \mathrm{~nm}$ thick PEDOT:PSS/graphene layer, which can be seen in the observed SPP dispersion.

\subsection{Ellipsometer measurements}

For spray-coated samples, the ellipsometric model used to fit the VASE data utilized uniaxial anisotropy with the perpendicular and parallel components using multiple Lorenz functions [33]. In 

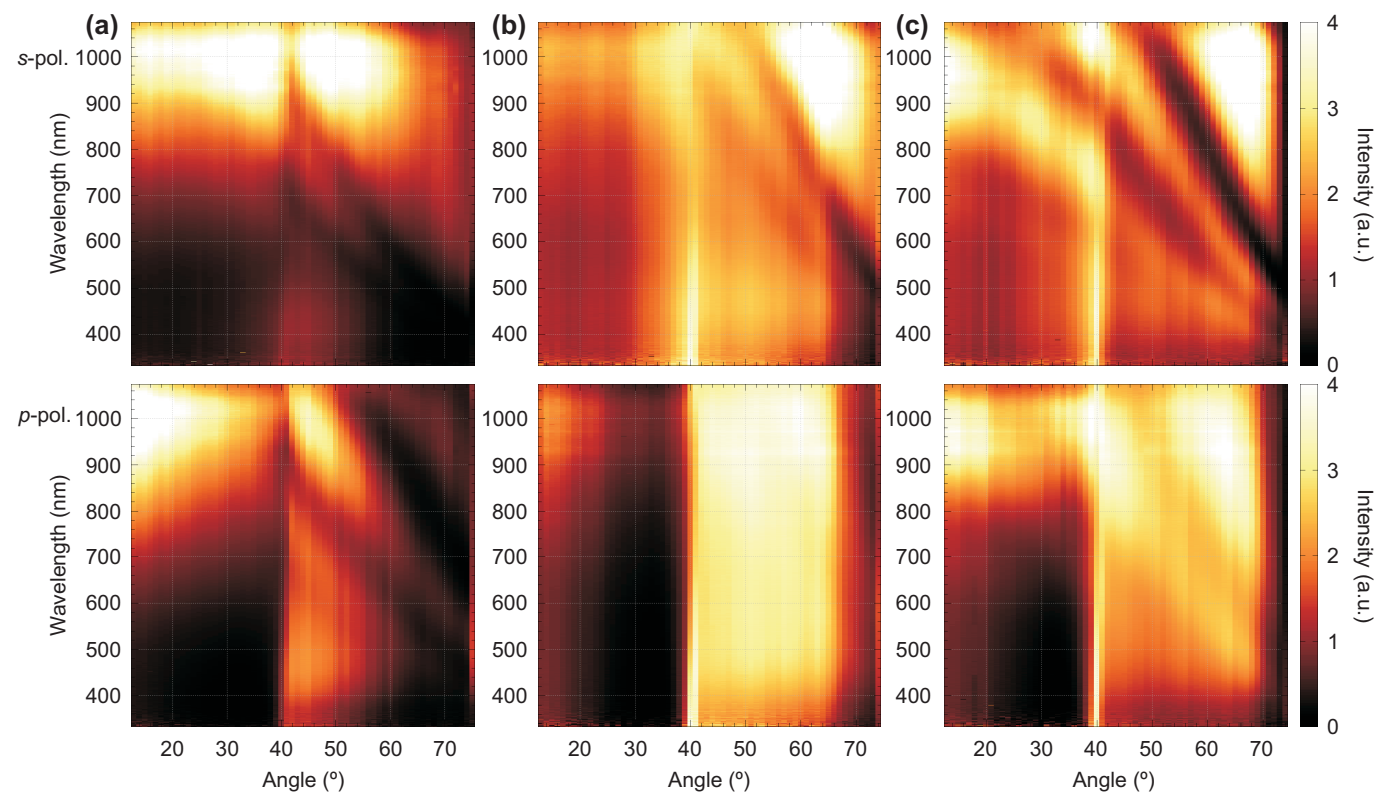

Figure 3: Reflection spectra for spray-coated films measured in total internal reflection conditions. (a) PEDOT:PSS, $500 \mathrm{~nm}$ thick (A-3), (b) PEDOT:PSS/graphene, $550 \mathrm{~nm}$ thick (B-4), (c) PEDOT:PSS/graphene, $560 \mathrm{~nm}$ thick, sonicated $90 \mathrm{~min}$ to reduce flake size (C-1). Upper row s-polarization and lower row p-polarization. Intensity of spectra below $41^{\circ}$ angles are not to scale.

cases where different thicknesses of the same material were measured, a coupled model has been used. The ellipsometry fits of the spin-coated samples were done with a model that allowed $n$ and $k$ values to vary independently. Fig. 4 shows the fitted real parts $n$ and imaginary parts $k$ of the refractive index in both horizontal and vertical directions for the PEDOT:PSS and PEDOT:PSS/graphene samples. The results show clear anisotropy in all the cases, but the effect of graphene is only minor. Note: the displayed curves have been smoothed using a cubic function to remove random noise in the calculated data.

The fitted functions of the refractive indices reveal that PEDOT:PSS films that include graphene have a slightly different optical response (Fig. 4a,b), but the difference is not significant based on ellipsometry measurements. Spray coating, like spin coating, also produces highly anisotropic films, contrary to earlier assumptions [22]. Even though ellipsometry is not very reliable in the case of a thick and rough spray-coated sample, anisotropy in the films is clearly visible here (Fig. 4c, Fig. S9 in Supplementary material).

For very thin (spin-coated) samples the mean square error (MSE) of the ellipsometry fits is 1.7 (Fig. 4a) and 1.4 (Fig. 4b), which is a good fit. For thick (spray-coated) samples the MSE is higher, of the order 20. In both cases the general shape and trend of the resulting material parameters are reasonably stable.

\subsection{Simulations}

To compare ellipsometric measurements with reflectometry, the reflection spectra were simulated using Comsol Multiphysics 5.0, a commercial implementation of the finite-element method. The developed model calculates the reflectance of an infinite plane wave incident on the air/film/glass interface. The model was reduced to a $2 \mathrm{D}$ geometry to speed up the calculation.

The simulation domain was $0.1 \mu \mathrm{m}$ by $6.5 \mu \mathrm{m}$ and contained 644 mesh elements. 12 hours of calculation time was used to generate the data in Fig. 5 on a cluster using 48 Intel Xeon X5650 processors. The resolution of the images in Fig. 5 is $201 \times 131$ points (wavelength and angle, respectively). The anisotropic refractive indices needed for the simulations were obtained from spectroscopic ellipsometry measurements.

The results were verified in the case of $s$-polarized light by comparing them to isotropic simulations performed with the transfer matrix method that we implemented with Matlab. This is possible because the $s$-polarized incident light only experiences the refractive index component parallel to the film.

Uniaxial anisotropy can be seen as shifting of the thin-film interference fringes. At $p$-polarization the effective refractive index is higher because the perpendicular direction component of the refractive index affects this polarization. This results in the decrease of distance between the fringes at a certain incidence angle compared to $s$-polarization, and the 

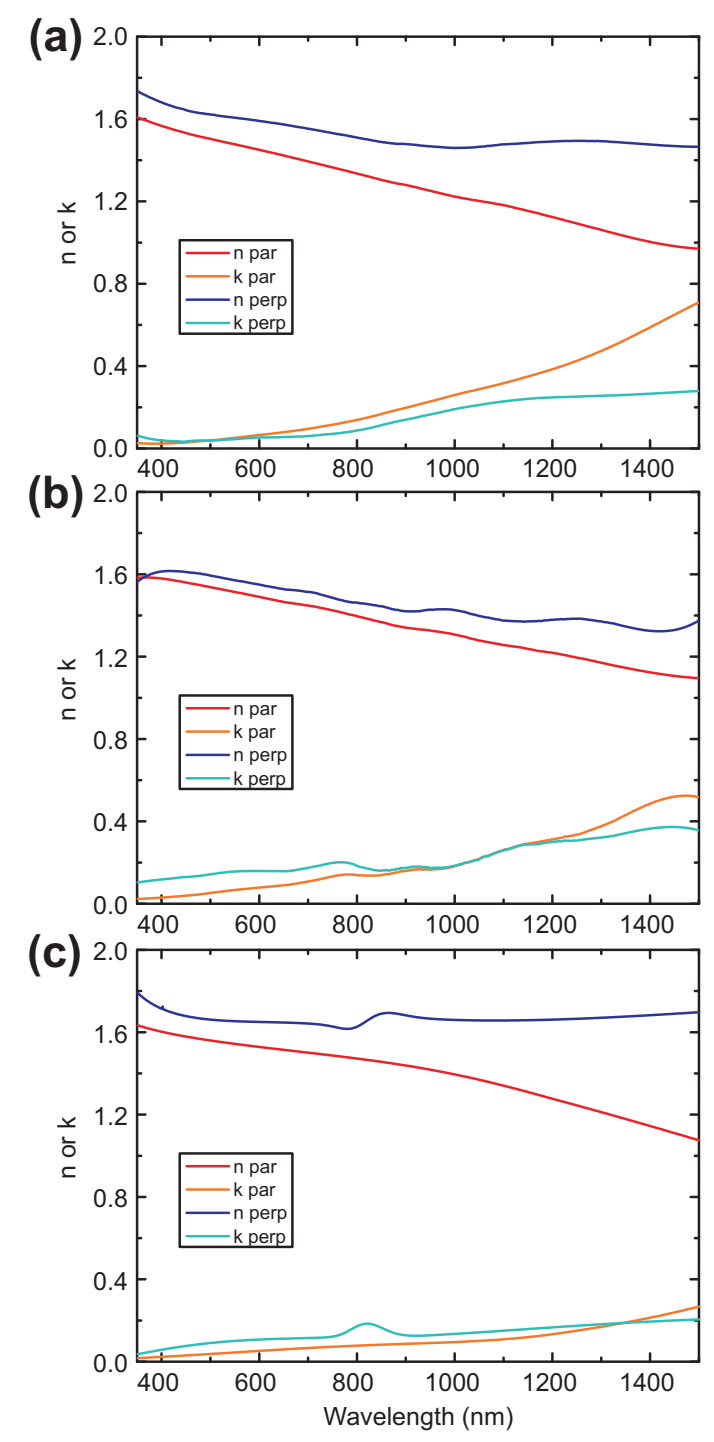

Figure 4: Refractive indices $n$ and extinction coefficients $k$ for both parallel and perpendicular directions of thin films acquired from ellipsometry fits. (a) Spin-coated PEDOT:PSS (combined result from samples A-S1 and A-S2). (b) Spin-coated PEDOT:PSS/graphene (only B-S1). (c) Spray-coated PEDOT:PSS/graphene, sonicated for 90 min (combined result from six samples, C-1 to C-6). movement of specific maxima and minima to longer wavelengths [30]. The change in refractive index as a function of wavelength can be seen in the curvature of the interference fringes.

For spray-coated samples the simulations (Fig. 5) match measurements (Fig. 3) reasonably well in terms of locations of the interference resonances. Inconsistencies are mostly due to the roughness and relatively high absorbance of the thin films, which produce difficulties in getting reliable ellipsometry data. This reveals the power of reflective measurements in this case.

\section{Conclusions}

Using variable-angle total internal reflection spectroscopy, we have found that spray-coated films of PEDOT:PSS on glass have a high degree of optical anisotropy at longer wavelengths in the infrared region. For spin-coated thin PEDOT:PSS films the anisotropic structure has been previously thought to have been caused by the spin-coating process, which would align the PSS-rich regions of high conductivity along the film plane [22]. Our measurements show that an alternative mechanism produces anisotropy in spray-coated films of all measured thicknesses. Previous anisotropy measurements with ellipsometry have only been done on sub-200 nm films, while our TIR measurements show clear anisotropic features in interference up to thickness of $2.5 \mu \mathrm{m}$. The addition of graphene to PEDOT:PSS greatly increases the conductivity of the films, but the optical anisotropy shows only minor enhancement on the thick films. The refractive index functions do not show significant differences between measured inks with and without graphene. TIR absorbance measurements show a clear advantage over ellipsometry in assessing thin film anisotropy in cases where the film is thick or uneven. Optically and electrically anisotropic graphene/PEDOT:PSS films have a wide range of applications in electronics, photonics and optoelectronics.

\section{Acknowledgement}

Funding from the Academy of Finland is gratefully acknowledged (Projects 135193, 218182, 263868, 263526, 283011, 138146). We thank Niko-Ville Hakkola for help in the reflection measurements. T.I. thanks the Finnish National Doctoral Programme in Nanoscience and the Vilho, Yrjö and Kalle Väisälä Fund of the Finnish Academy of Sciences and Letters for financial support. 

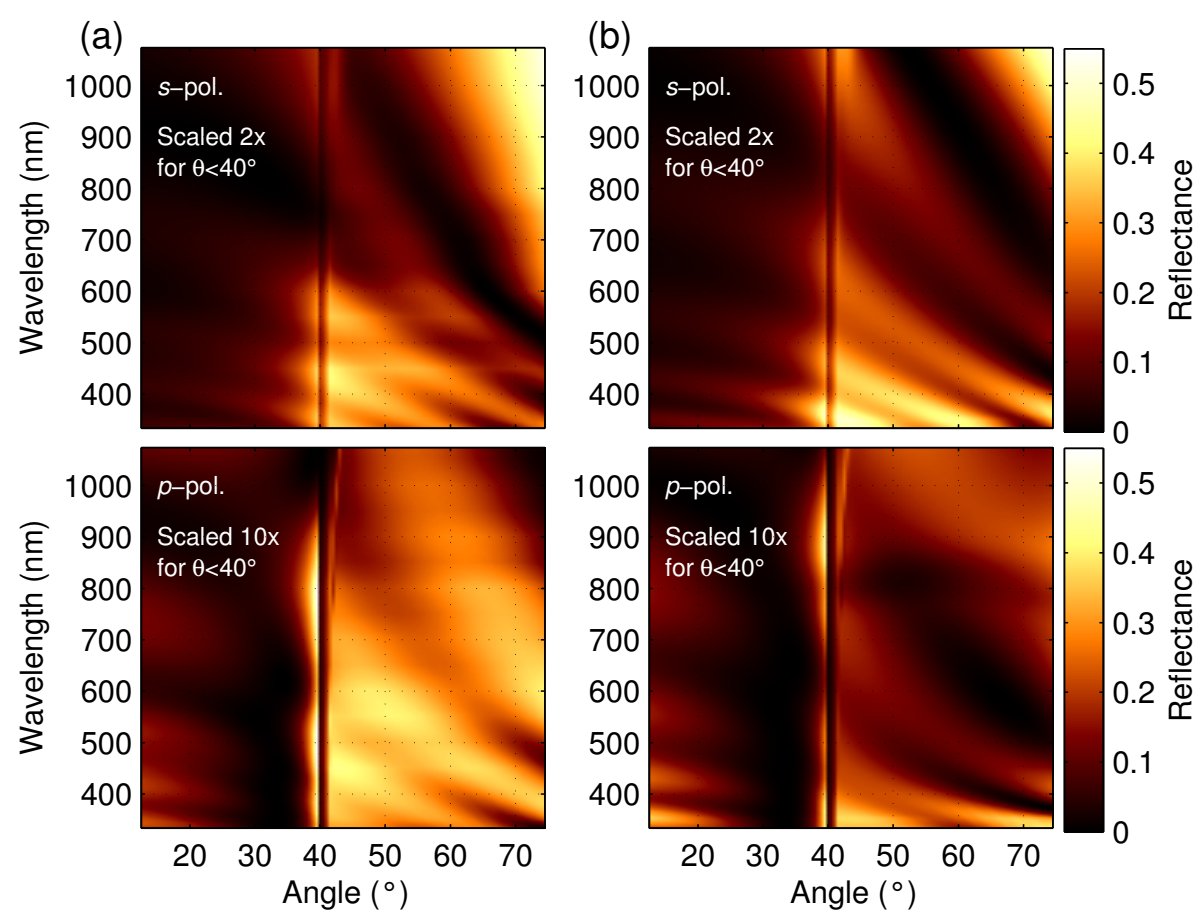

Figure 5: Reflection spectra in total internal reflection conditions, simulated from ellipsometry data. (a) PEDOT:PSS, $500 \mathrm{~nm}$ thick, refractive index from spin-coated samples [22]. (b) $560 \mathrm{~nm}$ thick PEDOT:PSS/graphene, uses data from Fig. 4c.

\section{References}

[1] A. K. Geim and K. S. Novoselov. The rise of graphene. Nat. Mater., 6:183-191, 2007.

[2] Konstantin S Novoselov, V I Fal, L Colombo, P R Gellert, M G Schwab, K Kim, et al. A roadmap for graphene. Nature, 490(7419):192200, 2012.

[3] John Robertson. Growth of nanotubes for electronics. Mater. Today, 10(1):36-43, 2007.

[4] S. Park, M. Vosguerichian, and Z. Bao. A review of fabrication and applications of carbon nanotube film-based flexible electronics. Nanoscale, 5(5):1727-1752, 2013.

[5] P. Avouris, Z. Chen, and V. Perebeinos. Carbon-based electronics. Nat. Nanotechnol., 2:605-615, 2007.

[6] Francesco Bonaccorso, Z Sun, T Hasan, and A C Ferrari. Graphene photonics and optoelectronics. Nat. Photonics, 4(9):611-622, 2010.

[7] P. Avouris, M. Freitag, and V. Perebeinos. Carbon-nanotube photonics and optoelectronics. Nat. Photonics, 2:341-350, 2008.

[8] D. R. Paul and L. M. Robeson. Polymer nanotechnology: Nanocomposites. Polymer, 49(15):3187-3204, 2008.

[9] Changgu Lee, Xiaoding Wei, Jeffrey W. Kysar, and James Hone. Measurement of the elastic properties and intrinsic strength of monolayer graphene. Science, 321(5887):385-388, 2008.

[10] M. F. L. De Volder, S. H. Tawfick, R. H.
Baughman, and A. J. Hart. Carbon nanotubes: Present and future commercial applications. Science, 339:535-539, 2013.

[11] A. N. Grigorenko, M. Polini, and K. S. Novoselov. Graphene plasmonics. Nat. Photonics, 6:749-758, 2012.

[12] Tommi Isoniemi, Andreas Johansson, J. Jussi Toppari, and Henrik Kunttu. Collective optical resonances in networks of metallic carbon nanotubes. Carbon, 63:581-585, 2013.

[13] Stephen R Forrest. The path to ubiquitous and low-cost organic electronic appliances on plastic. Nature, 428(6986):911-918, 2004.

[14] K Hecker and S. Breitung. OE-A Roadmap for Organic and Printed Electronics: White Paper, 5th ed., Organic Electronics Association (OEA). VDMA Verlag GmbH, 2013.

[15] Towards Green Electronics in Europe, The Strategic Research Agenda (SRA) for the Organic and Large Area Electronics (OLAE). Published on the OPERA website on September 18, 2009.

[16] Hyun-Jung Choi, Sun-Min Jung, Jeong-Min Seo, Dong Wook Chang, Liming Dai, and JongBeom Baek. Graphene for energy conversion and storage in fuel cells and supercapacitors. Nano Energy, 1(4):534-551, 2012.

[17] Suvi Lehtimäki, Sampo Tuukkanen, Juho Pörhönen, Pasi Moilanen, Jorma Virtanen, Mari Honkanen, and Donald Lupo. Low-cost, 
solution processable carbon nanotube supercapacitors and their characterization. Appl. Phys. A, 117(3):1329-1334, 2014.

[18] Andreas Elschner, Stephan Kirchmeyer, Wilfried Lovenich, Udo Merker, and Knud Reuter. PEDOT: Principles and applications of an intrinsically conductive polymer. CRC Press, 2010.

[19] S Timpanaro, Martijn Kemerink, F J Touwslager, M M De Kok, and S Schrader. Morphology and conductivity of pedot/pss films studied by scanning-tunneling microscopy. Chem. Phys. Lett., 394(4):339-343, 2004.

[20] T Hiate, N Miyauchi, Q Liu, R Ishikawa, $\mathrm{K}$ Ueno, and $\mathrm{H}$ Shirai. Real-time measurement of optical anisotropy during film growth using a chemical mist deposition of poly $(3,4-$ ethylenedioxythiophene):poly(styrenesulfonate). J. Appl. Phys., 115(12):123514, 2014.

[21] Tomohisa Ino, Taiga Hiate, Takeshi Fukuda, Keiji Ueno, and Hajime Shirai. Realtime ellipsometric characterization of the initial growth stage of $\operatorname{poly}(3,4-$ ethylenedioxythiophene):poly(styrene sulfonate) films by electrospray deposition using n,n-dimethylformamide solvent solution. J. Non-Cryst. Solids, 358(17):2520-2524, 2012.

[22] Leif A A Pettersson, Soumyadeb Ghosh, and Olle Inganäs. Optical anisotropy in thin films of poly (3, 4-ethylenedioxythiophene)-poly (4styrenesulfonate). Org. Electron., 3(3):143$148,2002$.

[23] Qiming Liu, Takashi Imamura, Taiga Hiate, Ishwor Khatri, Zeguo Tang, Ryo Ishikawa, Keiji Ueno, and Hajime Shirai. Optical anisotropy in solvent-modified poly $(3,4-$ ethylenedioxythiophene):poly(styrenesulfonic acid) and its effect on the photovoltaic performance of crystalline silicon/organic heterojunction solar cells. Appl. Phys. Lett., 102(24):243902, 2013.

[24] Zhipei Sun, Tawfique Hasan, Felice Torrisi, Daniel Popa, Giulia Privitera, Fengqiu Wang, Francesco Bonaccorso, Denis M Basko, and Andrea C Ferrari. Graphene mode-locked ultrafast laser. ACS nano, 4(2):803-810, 2010.

[25] Wenjing Hong, Yuxi Xu, Gewu Lu, Chun Li, and Gaoquan Shi. Transparent graphene/PEDOT-PSS composite films as counter electrodes of dye-sensitized solar cells. Electrochem. Commun., 10(10):1555-1558, 2008.

[26] Dohyuk Yoo, Jeonghun Kim, and Jung Hyun Kim. Direct synthesis of highly conductive poly (3, 4-ethylenedioxythiophene): poly (4-styrenesulfonate)(PEDOT: PSS)/graphene composites and their applications in energy harvesting systems. Nano Res., 7(5):717-730, 2014.

[27] Sampo Tuukkanen, Maija Hoikkanen, Minna Poikelispää, Mari Honkanen, Tiina Vuorinen, Markus Kakkonen, Jyrki Vuorinen, and Donald Lupo. Stretching of solution processed carbon nanotube and graphene nanocomposite films on rubber substrates. Synthetic Met., 191:28-35, 2014.

[28] Sampo Tuukkanen, Tuomas Julin, Ville Rantanen, Mari Zakrzewski, Pasi Moilanen, Kaisa E. Lilja, and Satu Rajala. Solution-processible electrode materials for a heat-sensitive piezoelectric thin-film sensor. Synthetic Met., 162(21):1987-1995, 2012.

[29] Tiina Vuorinen, Mari Zakrzewski, Satu Rajala, Donald Lupo, Jukka Vanhala, Karri Palovuori, and Sampo Tuukkanen. Printable, transparent, and flexible touch panels working in sunlight and moist environments. Adv. Funct. Mater., 24(40):6340-6347, 2014.

[30] G I Surdutovich, J Kolenda, J F Fragalli, L Misoguti, R Vitlina, and V Baranauskas. An interference method for the determination of thin film anisotropy. Thin Solid Films, 279(1):119-123, 1996 .

[31] P Weightman, D S Martin, R J Cole, and T Farrell. Reflection anisotropy spectroscopy. Rep. Prog. Phys., 68(6):1251, 2005.

[32] Yanfei Xu, Yan Wang, Jiajie Liang, Yi Huang, Yanfeng Ma, Xiangjian Wan, and Yongsheng Chen. A hybrid material of graphene and poly (3, 4-ethyldioxythiophene) with high conductivity, flexibility, and transparency. Nano Res., 2(4):343-348, 2009.

[33] Leif A A Pettersson, Fredrik Carlsson, Olle Inganäs, and Hans Arwin. Spectroscopic ellipsometry studies of the optical properties of doped poly (3,4-ethylenedioxythiophene): an anisotropic metal. Thin Solid Films, 313:356$361,1998$. 